\title{
Conexões
}

\section{Tradução e validação da escala "Orientation Toward a Sporting Event" (OSE) e sua utilização para a paralisação e segurança de eventos esportivos em meio a pandemia COVID-19}

\author{
Leandro Mazzei ${ }^{1}$ \\ Laura Carraro Costa ${ }^{1}$ \\ Sofia Camanho Ferreira ${ }^{1}$ \\ Luiz Eduardo Gaio ${ }^{1}$
}

\section{RESUMO}

O objetivo deste artigo foi traduzir e validar uma escala sobre as Motivações e os Valores que levam as pessoas aos eventos esportivos, assim como um melhor entendimento sobre os impactos da pandemia COVID-19 e a paralisação / segurança dos eventos esportivos neste contexto. Metodo: Foi utilizada a escala Orientation Toward a Sporting Event (OSE), adicionando-se perguntas sobre a Paralisação e a Segurança do público em eventos esportivos. Também foram realizadas perguntas sobre como os eventos esportivos devem ser em 2020 quanto a presença ou não de público. Para a análise dos dados, foi utilizada a técnica de Modelagem de Equações Estruturais e estatística descritiva. Resultados e discussão: Após respostas de 441 pessoas, concluiu-se que são significativas as relações entre os comportamentos Sociais e Afetivos do público para com o tema Paralisação. Também demonstrou-se ser significante a relação entre socialização e Segurança para a realização de eventos esportivos no contexto pandêmico. Conclusão: Os impactos negativos serão enormes, tanto econômicos, como sociais. Todos os envolvidos com o Esporte e os seus eventos, desde as organizações responsáveis, o poder público, o setor privado, até a mídia que os transmitem, deverão buscar possíveis soluções para sanar tais impactos negativos.

Palavras-chave: Eventos Esportivos. Orientação. Comportamento. COVID-19.

\footnotetext{
${ }^{1}$ Universidade Estadual de Campinas.
}

\section{Correspondência:}

Leandro Mazzei. Faculdade de Ciências Aplicadas, Universidade Estadual de Campinas, Rua Pedro Zaccaria, 1300, Jardim Santa Luiza, CEP 13484350, Limeira, SP, Email: leandro.mazzei@fca.unicamp.br

Recebido em: 3 jun. 2020

Aprovado em: 14 ago. 2020 


\section{Translation and validation of the "Orientation Toward a Sporting Event" (OSE) scale and its use for the Shutdown and Security of sporting events in the COVID- 19 pandemic context}

\section{ABSTRACT}

Objective: The aim of this article was to translate and validate a scale on the Motivations and Values that toward people to sporting events, as well as a better understanding of the impacts of the pandemic COVID-19 and the Shutdown / Security of sporting events in this context. Methods: The Orientation Toward a Sporting Event (OSE) scale, added to questions about Shutdown and Public Safety at sporting events. Questions about how sporting events should be in 2020, regarding the presence or not of an audience were also asked. For data analysis, the Structural Equation Modeling approach and descriptive statistics were used. Results and discussion: After the responses of 441 people, it was concluded that the relationship between the Social and Affective behaviors are significant to the theme Shutdown. The relationship between socialization and Security for sport events in the pandemic context was also shown to be significant. Conclusion: The negative impacts will be enormous, both economic and social. Everyone involved with Sport and its events, from the responsible organizations, the public authorities, the private sector, to the media that broadcasts them, should seek possible solutions to remedy such negative impacts.

Keywords: Sport events. Orientation. Behavior. COVID-19.

\section{Traducción y validación de la escala "Orientation Toward a Sporting Event" (OSE) y su uso para el Cerramiento y la Seguridad de eventos deportivos en medio de la pandemia de COVID-19}

\section{RESUMEN}

Objetivo: El propósito de este artículo fue traducir y validar una escala sobre las Motivaciones y Valores que llevan a las personas a eventos deportivos, así como una mejor comprensión de los impactos de la pandemia COVID-19 y el Cerramiento / Seguridad de los eventos deportivos en este contexto. Metodo: La escala Orientation Toward a Sporting Event (OSE) se utilizó para el análisis, agregando preguntas sobre el Cerramiento y la Seguridad en eventos deportivos. Para el análisis de datos, se utilizó la técnica de Modelado de Ecuaciones Estructurales y estadísticas descriptivas. Resultados y discusión: Después de las respuestas de 441 personas, se concluyó que las relaciones entre los comportamientos Sociales y Afectivos del público hacia el tema Cerramiento son significativas. Conclusión: La relación entre socialización y Seguridad para eventos deportivos en el contexto de la pandemia también fue significativa. Todos los involucrados en el deporte y sus eventos, desde las organizaciones responsables, el gobierno, el sector privado, los medios que los transmiten, deben buscar posibles soluciones para remediar tales impactos negativos.

Palabras Clave: Eventos deportivos. Orientacion. Comportamiento. COVID-19. 


\section{INTRODUÇÃO}

Esta pesquisa proporciona uma reflexão, com evidências científicas, sobre os impactos no Esporte causados pela pandemia COVID-19. Ao mesmo tempo, também é uma oportunidade de valorizar o conhecimento científico, aspecto muitas vezes negligenciado pelas organizações responsáveis pelo esporte no Brasil. Desta forma, este artigo teve como objetivo a tradução e validação de uma escala relacionada com as Motivações e os Valores pessoais que levam cada indivíduo a se fazerem presentes em eventos esportivos, assim como um melhor entendimento sobre o comportamento do público com relação à pandemia do COVID-19 e a paralisação e segurança nos eventos esportivos neste contexto.

Eventos esportivos são considerados acontecimentos que se constituem fato de importância na vida das pessoas (POIT, 2013). Também para muitos autores, os eventos esportivos são parte de uma "engrenagem", ligada ao desenvolvimento esportivo, seja em termos de suas políticas ou para a própria evolução esportiva de praticantes e atletas (BÖHME, 2011; DE BOSSCHER et al., 2015). E ainda há, o ponto de vista econômico, tornando os eventos esportivos parte importante de uma Indústria do Esporte (MATTAR; MATTAR, 2013; PITTS; FIELDING; MILLER, 1994).

De fato, o Esporte como um todo apresenta números economicamente abundantes e ostensivamente impressionantes, apesar das fontes para o números não serem precisas (GAMMELSÆTER, 2020). Alguns números mostram que o esporte representa valores em torno de US \$ 700 bilhões por ano, ou $1 \%$ do Produto Interno Bruto (PIB) global, onde as receitas anuais com eventos esportivos crescem constantemente, de US\$ 58 bilhões em 2009 para US\$ 80 bilhões em 2014 (GAMMELS/ETER, 2020). No cenário brasileiro, os números são defasados, mas em uma publicação mais confiável, apresentou-se movimentações em torno de US\$ 12 milhões em 2000, o que correspondia na altura 1,97\% do PIB nacional e em 2010 essa quantia alcançou US $\$ 45$ bilhões (correspondente a 2,14\% do PIB nacional), demonstrando uma evolução ascendente do esporte na participação do PIB brasileiro (KASZNAR; GRAÇA, 2012).

De qualquer forma, os eventos esportivos fornecem um forte conteúdo / valor emocional para os interessados, pois envolvem motivações, valores, experiências, sentimentos, interações sociais, dentre outros e, portanto, ocupam um papel de destaque em suas vidas (FUNK et al., 2009). Sobre outro ponto de vista, representam uma importante manifestação do fenômeno Esporte, mas que necessitam de planejamento, coordenação e gerência de recursos para que uma qualidade seja atingida a fim de atender as expectativas das pessoas que possuem algum sentimento pelo Esporte (GILLESPIE; MCDONALD, 2015; MASTERMAN, 2014; PREUSS, 2007). 
Quase não se sabe ao certo, mas talvez a partir do final de 2019 e início de 2020, todos nós fomos inseridos e um cenário comparável a uma Guerra Mundial, onde o surto de COVID-19 (doença causada pelo corona vírus e que provoca Síndrome Respiratória Aguda Grave) teve um apelo emergencial nos sistemas globais de saúde, além de um efeito cascata em todos os aspectos da vida humana como a que conhecíamos até então (NICOLA et al., 2020). Destaca-se a extensão do surto com a Organização Mundial da Saúde (OMS) declarando emergência global em 30 de janeiro de 2020, muito pela característica de alta taxa de transmissão presente no vírus (CHEN et al., 2020). Daí para frente, na tentativa de "achatar a curva" de pacientes, governos aplicaram paralisações nas fronteiras, restrições de viagens, quarentena e restrição para aglomerações e contato entre pessoas, o que fatalmente envolveu práticas e eventos esportivos (GUERREIRO et al., 2020).

Em uma tentativa de entender o efeito da pandemia no esporte, diversos estudos vêm sendo publicados (GALLEGO et al., 2020; GUERREIRO et al., 2020; MOHR et al., 2020; TORESDAHL; ASIF, 2020). Os estudos vão desde protocolos para uma prática esportiva segura (seja com objetivo de saúde, lazer ou rendimento), mas também e principalmente sobre os impactos que a turbulência da COVID-19 estão provocando no Esporte, especificamente aos eventos esportivos, como por exemplo os Jogos Olímpicos e os Campeonatos Nacionais de Futebol.

Assim, agregando conhecimento sobre os impactos no Esporte causados pela pandemia COVID-19, este artigo apresenta a tradução e validação da escala intitulada "Orientation Toward a Sporting Event" (OSE), adicionando-se a opinião das pessoas sobre a paralisação e segurança dos eventos esportivos em meio ao contexto da crise pandêmica. Apesar deste artigo utilizar a escala OSE no contexto pandêmico, com sua tradução e validação, ela poderá ser usada posteriormente em outros contextos, auxiliando gestores e organizadores na compreensão do comportamento dos indivíduos quanto à presença em eventos esportivos. O que consequentemente pode proporcionar melhores ações e processos gerenciais que visam uma melhor organização e potencialização do atendimento das expectativas do público que se interessa em participar e/ou prestigiar os variados tipos de eventos esportivos. Importante agradecer e destacar a revista Conexões sobre a acessibilidade e compreensão para a divulgação de mais uma fonte de informação, o que proporciona conhecimento e entendimento sobre um problema complexo, onde não existem soluções simples e precipitadas, que provavelmente possuem interesses escusos e que não respeitam evidências científicas e tão pouco os valores esportivos e da própria vida humana.

\section{REFERENCIAL TEÓRICO}

$\mathrm{Na}$ literatura sobre o comportamento do consumidor, o conceito de "Orientação" se refere à inclinação específica de um indivíduo em relação à adoção 
de um comportamento previsível para um determinado ato de "consumo" (PONS; MOURALI; NYECK, 2006). Como destacado por Pons et al. (2006), para a constituição deste comportamento, destaca-se a importância da motivação e dos valores presentes em cada indivíduo.

O aspecto da motivação requer a capacidade de um determinado produto ou serviço (eventos esportivos, por exemplo) em incitar a busca por uma satisfação, oriunda de alguma necessidade específica das pessoas. Lembrando que as necessidades podem variar desde fisiológicas, de segurança, afetivos-sociais (pertencimento), autoestima, e autorrealização (MASLOW, 2016). Com base na hierarquia de necessidades de Maslow, os gestores podem identificar diferentes tipos de interesses por parte das pessoas, e assim, posteriormente podem direcionar melhor a organização dos eventos esportivos. Em geral, diferentes serviços esportivos se adaptam a diferentes categorias de necessidades (FUNK, DANIEL; ALEXANDRIS; MCDONALD, 2016). Funk et al. (2009) demonstraram que a socialização é um fator motivador para indivíduos participarem de jogos esportivos ou participarem de atividades esportivas em grupo (ou seja, satisfazem a necessidade de pertencer). Além disso, existem serviços esportivos que atendem a necessidades específicas do ego (autoestima). Por exemplo, os participantes de corridas de rua, competem entre si, mas geralmente possuem mais objetivos pessoais (terminar a prova ou atingir um tempo específico), o que os ajuda a satisfazer as necessidades individuais. Existem também serviços esportivos que atendem às necessidades de autoatualização. Foi sugerido que o aprendizado e o desenvolvimento de habilidades são os principais fatores motivadores para muitas pessoas praticarem esportes, como tênis, esqui e snowboard, pois propõe em suas práticas desafios individuais que proporcionam autoestima ao serem realizados (FUNK et al., 2009).

Já a dimensão "valores" é formada pelo conjunto de concepções formadas durante a vida das pessoas, compactuadas a partir da família, amigos, ambientes em que os indivíduos passam ao longo de sua existência (WANN et al., 2008). De fato, os valores direcionam o comportamento das pessoas sobre um determinado contexto social, produto e até serviço (PONS; MOURALI; NYECK, 2006).

Assim, o componente de motivação refere-se à capacidade do evento de satisfazer um indivíduo quanto às necessidades e desejos de fazer parte de um grupo, presenciar momentos que farão parte da memória afetiva, o desejo de expressar a alegria, o desejo de fazer parte ou compartilhar sentimentos em um ambiente social, dentre outros. A noção de afiliação de grupo é apresentada na literatura de marketing esportivo como uma das principais razões para explicar altos níveis de envolvimento dos apaixonados pelo esporte (WANN et al., 2008).

No caso do componente de valores, geralmente também se manifestam quando o indivíduo precisa fazer uma escolha entre várias atividades de lazer ou entretenimento, e escolhe um evento esportivo específico, de acordo com seu 
sistema de valores. Os valores podem ser identificados através da escolha de um esporte em particular, ou também pela forma como o evento é vivenciado (PONS; MOURALI; NYECK, 2006). Alguns sinais e significados compartilhados entre o próprio grupo (rituais, roupas, símbolos, etc.), oferecem às pessoas a oportunidade de escolher e de expressar o seu comportamento e compartilhar valores (apaixonados, fanáticos, especialistas, calmos, barulhentos, gastadores, etc.) (WANN et al., 2008).

Pons et al. (2006) desenvolveram uma escala padronizada e validada com o propósito de proporcionar uma melhor compreensão sobre a "Orientação" das pessoas para eventos esportivos. A escala Orientation Toward A Sporting Event (OSE) busca entender a orientação dos indivíduos para eventos esportivos a partir de comportamentos Afetivos, Cognitivos e Sociais. Para cada uma dessas dimensões, existem cinco sentenças (totalizando 15), que são apresentadas aos respondentes que devem opinar em uma escala de Likert de cinco pontos, discordando ou concordando totalmente com uma determinada afirmação que corresponde, respectivamente, a um determinado comportamento.

A escala proporciona uma classificação do comportamento das pessoas, que podem ser "Super fãs", "Fãs Sociais", "Fãs Experenciais" e "Fãs Situacionais". Super Fãs são indivíduos com alta pontuação em cada uma das dimensões OSE (acima de 4,2 em média), esses indivíduos apresentam uma forte Orientação geral para eventos esportivos, ou seja, em tese comparecem/assistem a eventos esportivos com maior frequência. Fãs Sociais englobam indivíduos que são moderados na orientação geral, mas possuem médias superiores na dimensão Social sobre orientação aos eventos esportivos, ou seja, este tipo possui a motivação e valores para ir aos eventos esportivos e socializarem fortemente com outras pessoas. Fãs Experenciais descrevem indivíduos que também possuem orientação geral moderada (valores médios na escala), mas que possuem valores médios maiores na dimensão Afetiva, seu principal interesse no evento está diretamente ligado a emoção de estar ou assistir o acontecimento esportivo (experiência). Por fim, Fãs Situacionais ou Ocasionais representam indivíduos com a menor média nos valores considerando toda a escala. Fã Situacionais veem, escutam ou, até mesmo, falam sobre esporte, mas possuem baixo interesse com relação aos eventos esportivos, se fazendo presentes apenas em ocasiões importantes (PONS; MOURALI; NYECK, 2006).

A escala OSE tem sido utilizado principalmente para o dimensionamento de "grupos" de interessados em eventos esportivos, no sentido de que cada grupo possui características e expectativas específicas (BOUCHET et al., 2011). Também foi utilizada para medir a relação entre a orientação para os eventos esportivos e a auto-congruência (comportamento que envolve personalidade do indivíduo e circunstâncias sociais), personalidade da marca, patrocínio e comportamento de consumo esportivo, onde os resultados apontam que a orientação com base na auto-congruência em relação ao esporte e à personalidade da marca está 
positivamente associada aos resultados do patrocínio (PLEWA; PALMER, 2014).

As vantagens da escala OSE é que ela abrange as motivações dos indivíduos, supera as dificuldades encontradas na tentativa de medir a motivação, além de possuir o componente "valor" da teoria da orientação. Os valores específicos do contexto são estabelecidos com base no situacional e dependem disso para auxiliar no direcionamento do comportamento das pessoas. Portanto, o comportamento dominante, é resultado de contextos situacionais e ditado pelos valores mantidos pelos indivíduos (PLEWA; PALMER, 2014).

Desta forma, acompanhando o situacional, nada mais coerente de que adicionar à escala os efeitos da pandemia do COVID-19, principalmente no que tange os aspectos de segurança e das opiniões sobre a paralisação dos eventos esportivos. O contexto ligado à pandemia do COVID-19 trouxe inúmeros impactos para a vida humana, e as consequências sobre o Esporte e seus eventos não passaram imunes. A Paralisação dos eventos tem apoio do público no contexto pandêmico? As questões com relação à Segurança serão itens obrigatórios e imprescindíveis em eventos esportivos em uma nova realidade? Essas são questões decorrentes do ineditismo do qual a humanidade passa neste momento. Desta forma, este artigo apresenta mais informações sobre alguns impactos no contexto esportivo, especificamente neste caso, ao comportamento das pessoas com relação à presença em eventos esportivos, além de, possivelmente, a própria gestão e organização dos eventos esportivos daqui em diante.

\section{MÉTODO}

A presente pesquisa se caracteriza como descritiva, com abordagem quantitativa, uma vez que pretende expor características de determinado contexto (CRESWELL, 2010), especificamente sobre a "Orientação" das pessoas para os eventos esportivos, assim como a análise do comportamento do público com relação a pandemia do COVID-19 e a Paralisação e Segurança dos eventos esportivos. As pesquisas descritivas são um tipo pesquisa conclusiva que tem como principal objetivo descrição de algo, normalmente características ou funções presentes em um determinado universo amostral, mas ao mesmo tempo podem estabelecer correlações servindo como base para reflexões (MALHOTRA, 2011).

Para atingir seus objetivos e como forma de medir a orientação para eventos esportivos, foi utilizada a já citada escala Orientation Toward a Sporting Event (OSE), desenvolvida por Pons e colaboradores (2006). A escala OSE busca entender a Orientação dos indivíduos para eventos esportivos a partir de respostas/opiniões relacionadas com comportamentos Afetivos, Cognitivos e Sociais. Para cada um desses grupos, existem cinco afirmações (totalizando 15), que são apresentadas aos respondentes que devem opinar, em uma escala de 
Likert de cinco pontos, discordando ou concordando totalmente com cada sentença.

Conforme recomendado por Beanton et al. (2000), a escala foi devidamente traduzida por dois pesquisadores independentes. O objetivo é identificar as expressões e frases que apresentam desafios na tradução. Outra recomendação atendida, foi o fato de que o perfil dos pesquisadores tenha sido distinto, sendo um deles especialista no assunto abordado pela escala, e o outro sem conhecimento prévio, mas com fluência em inglês. Cada um produziu uma versão em português da escala que, posteriormente foram utilizadas para o desenvolvimento de um documento único, onde as inconsistências foram discutidas e decisões sobre a melhor opção (considerando o contexto brasileiro e semântica em português) foram tomadas.

Após a versão única, ainda foi realizado um pré-teste com algumas pessoas do grupo de pesquisas do qual os pesquisadores pertencem, com o objetivo de realizar a avaliação da redação e do entendimento dos itens do questionário (também chamada de validação de face) (PASQUALI, 2010). Após essa aplicação, a versão final pode ser visualizada abaixo (Quadro 1).

Quadro 1 - Escala Orientation Toward a Sporting Event (OSE) traduzida para o português

\begin{tabular}{|c|c|}
\hline & Escala Orientation Toward a Sporting Event (OSE) \\
\hline \multirow{5}{*}{$\begin{array}{l}\text { Dimensão } \\
\text { Afetiva }\end{array}$} & Para mim, ir em eventos esportivos é um verdadeiro prazer \\
\hline & Estou sempre animado quando vou a um evento esportivo \\
\hline & Fico sempre entusiasmado quando penso em ir em um evento esportivo \\
\hline & Quando eu vou em um evento esportivo, às vezes sinto que faço parte do evento \\
\hline & Sinto-me muito feliz quando posso ir em um evento esportivo \\
\hline \multirow{5}{*}{$\begin{array}{l}\text { Dimensão } \\
\text { Cognitiva }\end{array}$} & $\begin{array}{l}\text { Assistir a eventos esportivos me proporciona uma maior sensação de } \\
\text { proximidade com meus ídolos esportivos }\end{array}$ \\
\hline & Eu me considero um grande conhecedor de esportes \\
\hline & $\begin{array}{l}\text { Eu posso falar sobre táticas e estratégias esportivas igual aos comentaristas } \\
\text { esportivos profissionais }\end{array}$ \\
\hline & Sei muito pouco sobre esportes \\
\hline & $\begin{array}{l}\text { Eu realmente me interesso por qualquer informação sobre esportes (recordes, } \\
\text { números, transações, curiosidades) }\end{array}$ \\
\hline \multirow{5}{*}{$\begin{array}{l}\text { Dimensão } \\
\text { Social }\end{array}$} & Estou frequentemente envolvido em conversas sobre eventos esportivos \\
\hline & Eu gosto de falar sobre eventos esportivos com pessoas que conheço \\
\hline & $\begin{array}{l}\text { Assistir a um evento esportivo na TV é uma boa oportunidade para socializar } \\
\text { com os amigos }\end{array}$ \\
\hline & $\begin{array}{l}\text { Geralmente compartilho meus pensamentos e sentimentos sobre eventos } \\
\text { esportivos com outras pessoas }\end{array}$ \\
\hline & Participar de eventos esportivos é uma boa oportunidade para socializar \\
\hline
\end{tabular}


A partir da escala OSE, é possível classificar os respondentes em "Super fãs" (valor médio acima de 4,2 para todas as dimensões), "Fãs Sociais" (valor médio alto na dimensão Social, se comparado à média geral e das outras dimensões), "Fãs Experenciais" (valor médio alto na dimensão Afetiva, se comparado à média geral e das outras dimensões) e "Fãs Situacionais" (valor médio baixo para todas as dimensões) (PONS; MOURALI; NYECK, 2006).

Somadas à escala OSE, foram adicionadas perguntas ou sentenças relacionadas com aos eventos esportivos e a pandemia COVID-19, como por exemplo:

- "Você concorda que os eventos esportivos parem até o final de 2020? " (Paralisação). Com respostas em escala Likert de 1 a 5 entre discordo totalmente e concordo totalmente.

- "Se os eventos esportivos voltassem a ocorrer ainda esse ano, mas antes que um tratamento ou vacina fossem criados contra o COVID-19, como você se sentiria em comparecer aos eventos esportivos?" (Segurança). Com respostas em escala Likert de 1 a 5 entre nenhum pouco seguro e totalmente seguro.

Para essas duas dimensões, Paralisação e Segurança "de" e "em" eventos esportivos, não foram utilizadas escalas específicas ou de estudos prévios. Entretanto, para os conceitos ou valores relacionados à Paralisação e Segurança, utilizou-se sugestões de afirmações e itens da obra de Bruner II (2009), que contém uma série de exemplos psicométricos baseado em artigos publicados nos principais periódicos de marketing entre 2002 a 2005. Assim, para a Paralisação foi adaptada a escala que mede originalmente a opinião sobre "Fechamento de Empresa" e para Segurança, uma escala sobre a importância desta temática para as pessoas (BRUNER II, 2009). Também foram realizadas perguntas de opinião sobre, caso os eventos esportivos voltem a ser realizados em 2020, qual o formato quanto a presença ou não de público?

Participaram da pesquisa um grupo de 441 pessoas, que voluntariamente se dispuseram a responder formulário online divulgado em redes sociais, oriundas principalmente de comunidades da Universidade Estadual de Campinas, da cidade Campinas e região. O formulário ficou disponibilizado no período de 30 de abril de 2020 a 08 de maio de 2020, totalizando nove dias. Os respondentes receberam, de forma preliminar, as informações sobre o objetivo da pesquisa e atestaram termo de consentimento livre e esclarecido sobre a utilização dos dados de suas respostas (CAAE: 18344219.4.0000.5404). Somente os formulários completos foram considerados, apresentando então um perfil de respondentes comporto por 266 (60\%) homens e 175 (40\%) mulheres. Abaixo informações quanto a faixa etária dos respondentes (Figura 1). 


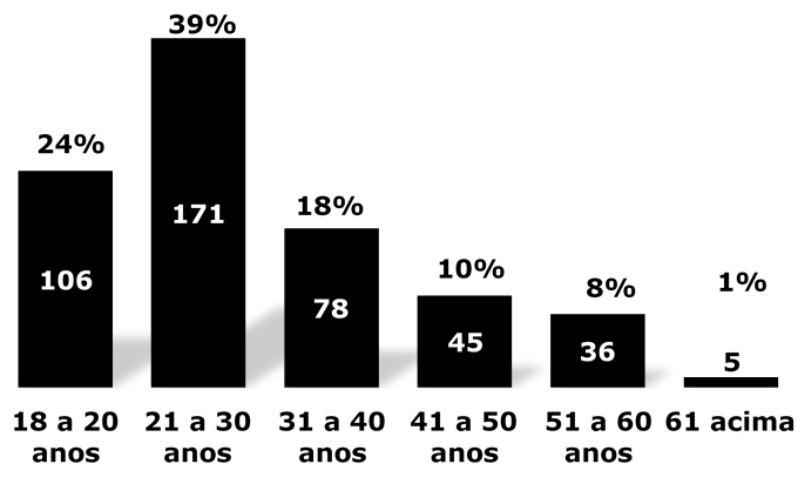

Figura 1 - Faixa etária dos respondentes.

Quanto ao grau de escolaridade, 227 (52\%) dos respondentes possuem curso superior completo, com ou sem pós-graduação, 191 (43\%) dos respondentes possuem nível superior incompleto e 23 (5\%) com ensino médio completo.

Para a análise dos dados, foi utilizada a técnica de Modelagem de Equações Estruturais (Structural Equation Modeling) baseada em variância (VB-SEM) com a utilização do software SmartPLS versão 3.2.4. O propósito foi analisar as relações entre os constructos, especificamente entre a escala OSE, orientação e opinião sobre a Paralisação do eventos esportivos devido a COVID-19 ("Você concorda que os eventos esportivos parem até o final de 2020?") e a Segurança do público com relação a presença em eventos esportivos ("Se os eventos esportivos voltassem a ocorrer ainda esse ano, mas antes que um tratamento ou vacina fossem criados contra o COVID-19, como você se sentiria em comparecer aos eventos esportivos?") como apresentado na Figura 2. O número amostral de 441 indivíduos viabiliza a realização de análises multivariadas e de Modelagem de Equações Estruturais do constructo (17 itens), pois cumpre a premissa de 10 respondentes para cada variável, conforme recomendado por Hair et al. (2009). Para a estimativa do modelo estrutural foi realizado a abordagem de boostraping, que realiza re-amostragens estatísticas buscando testar a significância do modelo. Para facilitar a convergência dos resultados, foram realizadas 5000 re-amostragens na estatística boostraping. 


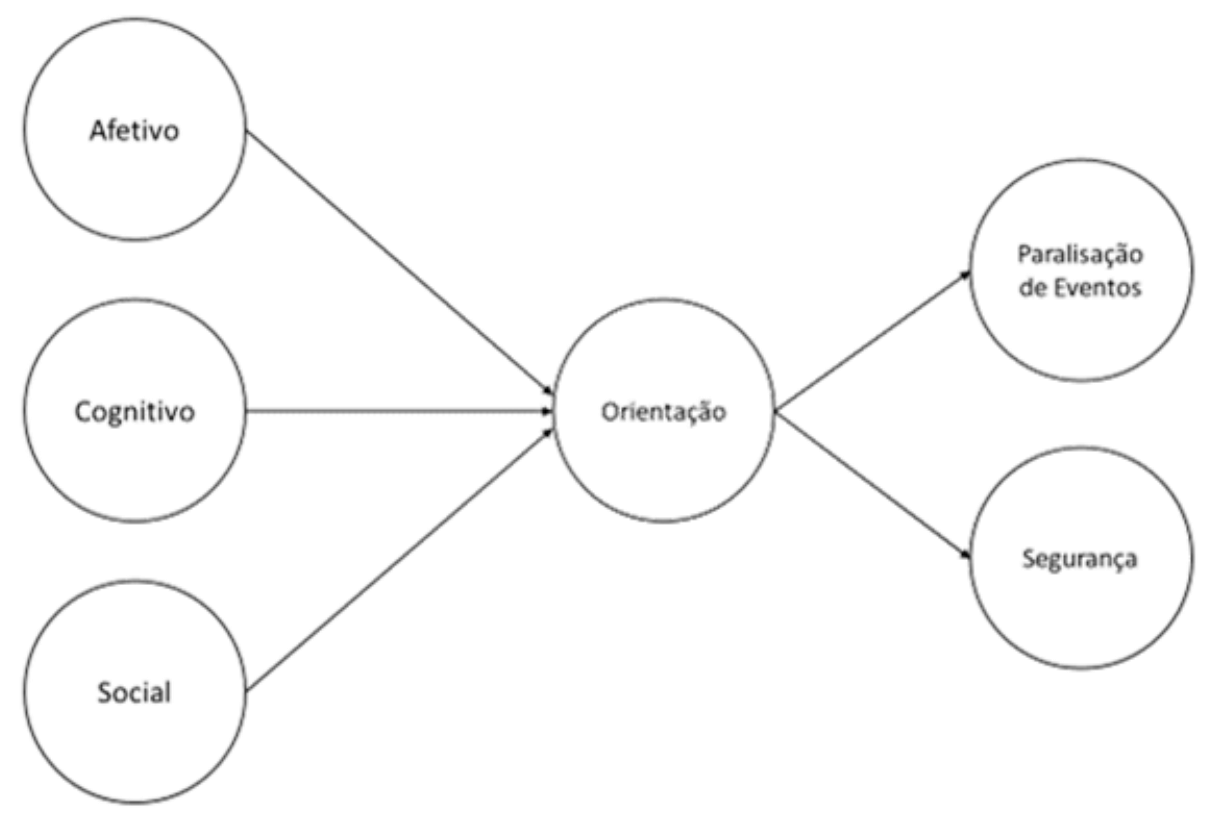

Figura 2 - Constructos e relações a serem verificadas entre eles.

Para verificar a confiabilidade doa escala e do modelo utilizado, foi utilizado o Alpha de Cronbach (HAIR et al., 2014). Além da Modelagem de Equações Estruturais, também foi utilizado estatística descritivas referente as outras questões/opiniões sobre os eventos esportivos e os impactos causados pela pandemia COVID-19.

\section{RESULTADOS E DiscussÃo}

Os resultados serão apresentados a partir da análise da classificação dos respondentes considerando os resultados da escala OSE; confiabilidade da escala utilizada; análise das relações entre as variáveis do constructo proposto e análise descritiva sobre as questões/opiniões sobre os eventos esportivos e os impactos causados pela pandemia COVID-19.

Assim, podem ser observados na Tabela 1, os números de respondentes classificados e os resultados médios para as dimensões da escala OSE, assim como e das perguntas sobre Paralisação e Segurança.

Tabela 1 - Resultados descritivos relacionados com a Escala OSE

\begin{tabular}{lcccc}
\hline & Super Fãs & Fãs Sociais & Fãs Experenciais & Fãs Situacionais \\
\hline Respondentes & $\mathbf{1 9 5}$ & $\mathbf{1 1 8}$ & $\mathbf{5 4}$ & $\mathbf{7 4}$ \\
AFETIVO & 4,86 & 4,22 & $\mathbf{4 , 4 9}$ & 2,91 \\
COGNITIVO & 3,71 & 3,03 & 2,87 & 2,44 \\
SOCIAL & 4,78 & $\mathbf{4 , 3 4}$ & 3,49 & 2,74 \\
ORIENTAÇÃO & $\mathbf{4 , 4 5}$ & 3,86 & 3,61 & $\mathbf{2 , 6 9}$ \\
PARALISAÇÃO & 3,10 & 3,39 & 3,76 & 3,89 \\
SEGURANÇA & 2,21 & 1,92 & 1,65 & 1,64 \\
\hline
\end{tabular}


Ainda pode ser visualizado na Tabela 1 valores acima de 3,00 para a opinião sobre a Paralisação e valores abaixo de 2,5 sobre a Segurança dos eventos esportivos em tempos de pandemia. Lembrando que para a Paralisação, os respondentes deveriam opinar em uma escala de Likert de 5 pontos entre "discordo totalmente" e "concordo totalmente", e para a Segurança, uma mesma escala Likert de 5 pontos, mas variando de "pouco seguro" para "totalmente seguro."

Sobre a confiabilidade da escala e resultados, o Alfa de Cronbach ficou entre 0,70 e 0,95 para as dimensões e ligação entre o constructo, com exceção da dimensão cognitivo que ficou inferior. No entanto, por utilizar uma escala previamente estabelecida, optou-se por não alterar as variáveis.

Para realizar a validade dos constructos é necessário analisar a validade convergente e validade discriminante, conforme descreve Hair et al. (2014). As Tabelas 2 e 3 apresentam as estatísticas necessárias para a validade convergente.

Tabela 2 - Correlação de Fornell-Larcker e estatísticas de validade

\begin{tabular}{lccccc}
\hline & AFETIVO & COGNITIVO & PARALISAÇÃO & SEGURANÇA & SOCIAL \\
\hline AFETIVO & $\mathbf{0 . 8 7 7}$ & & & & \\
COGNITIVO & 0.654 & $\mathbf{0 . 7 8 6}$ & & & \\
PARALISAÇÃO & -0.226 & -0.287 & $\mathbf{1 . 0 0 0}$ & & \\
SEGURANÇA & 0.176 & 0.189 & -0.445 & $\mathbf{1 . 0 0 0}$ & \\
SOCIAL & 0.726 & 0.774 & -0.280 & 0.218 & $\mathbf{0 . 7 8 3}$ \\
\hline Alfa de Cronbach & 0.924 & 0.368 & 1.000 & 1.000 & 0.845 \\
rho_A & 0.935 & 0.857 & 1.000 & 1.000 & 0.905 \\
Confiabilidade composta & 0.943 & 0.736 & 1.000 & 1.000 & 0.885 \\
Variância Média Extraída & & & & & \\
(AVE) & 0.768 & 0.618 & 1.000 & 1.000 & 0.613 \\
\hline
\end{tabular}

Para a validade convergente na Tabela 2, se reporta os resultados da confiabilidade composta, também denominada de Confiabilidade do Constructo. Para as 5 dimensões e suas variáveis latentes analisadas os valores foram superiores a 0,7, conforme recomenda Hair et al. (2009). Outra estatística utilizada refere-se média da Variância Extraída (AVE), que devem ser superiores a 0,5 , para validar os constructos. Os resultados da $A V E$, reportados na Tabela 2 são superiores todos válidos $(>0,5)$.

Hair et al. (2014) afirma que a validade discriminante é o grau em que uma dimensão é verdadeiramente diferente dos demais. O que oferece evidência de que uma dimensão é única e captura alguns fenômenos que outras medidas não conseguem. Para a validade discriminante foi analisado as correlações entre as dimensões, também denominada de Correlação de Fornell-Larcker. Os resultados são válidos quando os valores das correlações diagonais são superiores aos demais valores de cada dimensão. Os resultados da estatística de Fornell-Larcker 
reportados na Tabela 2, em destaque, indicam que o constructo é valido, já que suas correlações são superiores e próximas de 1 .

As cargas fatoriais cruzadas estão reportados na Tabela 3. Os valores destacados representam as cargas das variáveis de cada dimensão. Nota-se que todos os valores destacados são superiores a 0,5 e a maioria superiores a 0,7,0 que valida a convergência das variáveis e dimensões, como aponta Hair et al. (2014). Vale destacar também que as cargas fatoriais, em destaque, das variáveis de cada dimensão são superiores às cargas cruzadas quando se as comparam em outra dimensão.

Tabela 3 - Cargas fatoriais e cargas cruzadas

\begin{tabular}{|c|c|c|c|c|c|}
\hline & AFETIVO & COGNITIVO & SOCIAL & PARALISAÇÃO & SEGURANÇA \\
\hline ANIMADO & 0.912 & 0.592 & 0.659 & -0.24 & 0.152 \\
\hline ENTUSIASMADO & 0.909 & 0.573 & 0.643 & -0.211 & 0.148 \\
\hline FELIZ & 0.878 & 0.568 & 0.622 & -0.142 & 0.127 \\
\hline IR_EVENTOS & 0.894 & 0.611 & 0.693 & -0.21 & 0.179 \\
\hline VEJO_EVENTO & 0.782 & 0.514 & 0.551 & -0.164 & 0.162 \\
\hline CONHECEDOR_ESPORTE & 0.537 & 0.89 & 0.681 & -0.267 & 0.158 \\
\hline PROXIMIDADE_IDOLOS & 0.556 & 0.599 & 0.512 & -0.171 & 0.119 \\
\hline SEI_POUCO & -0.473 & -0.796 & -0.577 & 0.254 & -0.144 \\
\hline ESTRATEGIA_ESPORTIVA & 0.465 & 0.829 & 0.62 & -0.21 & 0.143 \\
\hline INTERESSO_INFORMACOES & 0.563 & 0.787 & 0.643 & -0.21 & 0.174 \\
\hline ENVOLVIMENTO_EVENTO & 0.578 & 0.698 & 0.88 & -0.28 & 0.234 \\
\hline EVENTO_TV & 0.454 & 0.457 & 0.552 & -0.021 & 0.076 \\
\hline FALAR_EVENTO & 0.651 & 0.715 & 0.903 & -0.27 & 0.195 \\
\hline PARTICIPAR_EVENTOS & 0.628 & 0.482 & 0.688 & -0.173 & 0.146 \\
\hline PENSAMENTOS & 0.571 & 0.64 & 0.834 & -0.213 & 0.139 \\
\hline PARAR_2020 & -0.226 & -0.287 & -0.28 & 1 & -0.445 \\
\hline VOLTAR_EVENTO & 0.176 & 0.189 & 0.218 & -0.445 & 1 \\
\hline
\end{tabular}

Nota: Cargas fatoriais em destaque.

A validade convergente de todas as variáveis, pelas três estatísticas, além de corroborar com o estudo de Pons et al. (2006), permitiu que não houvesse necessidade de nenhum ajuste nas variáveis. Todos esses resultados até aqui apresentados, proporcionam que essa escala seja utilizada em outros estudos e em sua versão em português.

Os resultados do modelo estrutural estão reportados na Figura 3. 


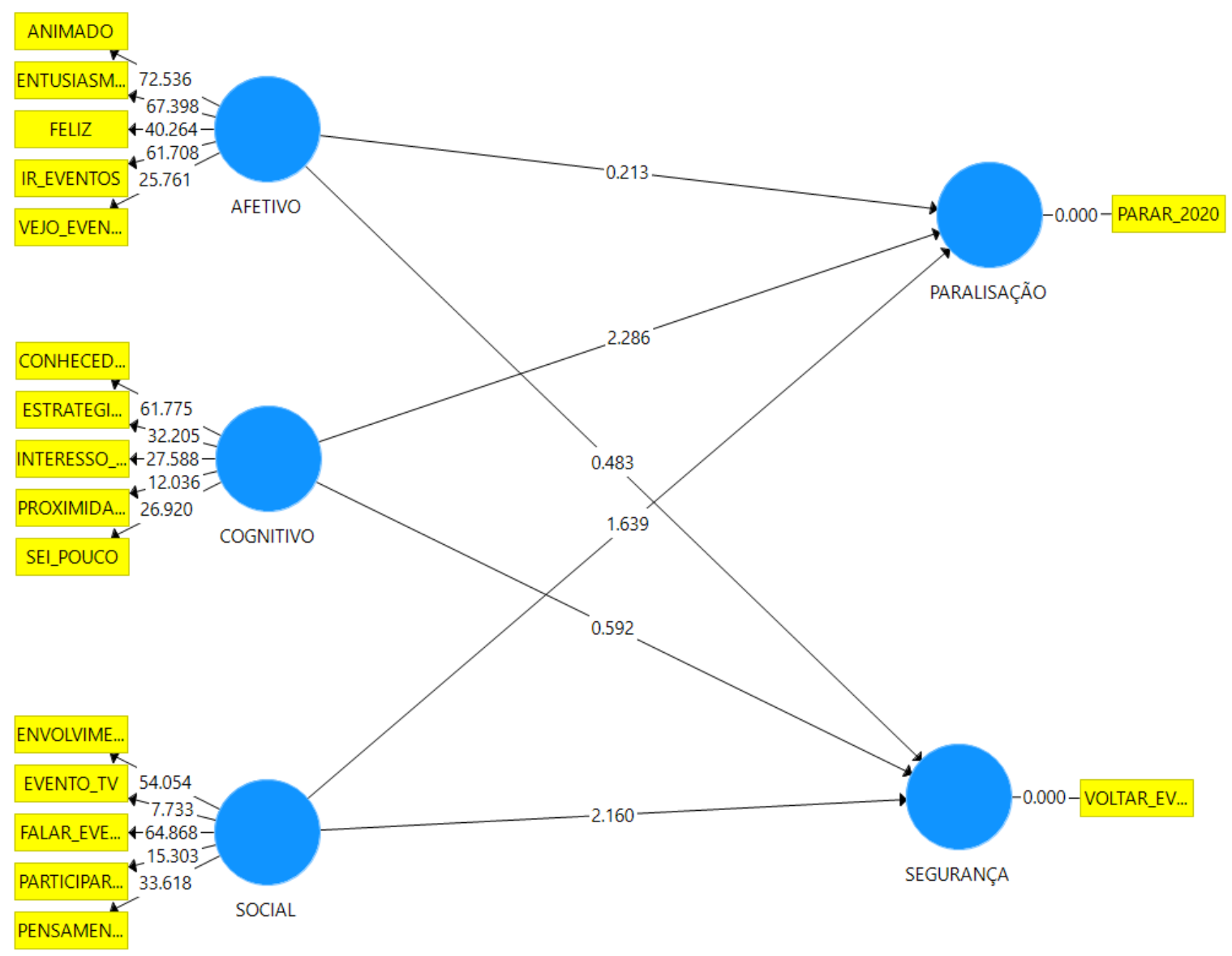

Figura 3 - Resultado da Modelagem Estrutural realizada no constructo proposto.

Os resultados da estatística VIF (Variance inflator fator), que mensura o efeito de multicolinearidade dos dados, são apresentados na Tabela 5, onde se indica que as regressões estruturais não apresentam colinearidade entre as variáveis, uma vez que os valores foram inferiores a 5 , como recomenda Hair et al. (2014).

Tabela 5 - Resultado Estatísticos da Modelagem Estrutural realizada

\begin{tabular}{lccccccc}
\hline & VIF & $\mathbf{f}^{\mathbf{2}}$ & Coef. & $\begin{array}{c}\text { Erro } \\
\text { Padrão }\end{array}$ & Est. t & Valor-p & $\begin{array}{c}\mathbf{R}^{\mathbf{2}} \\
\text { Ajust. }\end{array}$ \\
\hline AFETIVO -> PARALISAÇÃO & 2.212 & 0.000 & -0.014 & 0.067 & 0.213 & 0.83 & \\
COGNITIVO -> PARALISAÇÃO & 2.613 & 0.012 & -0.171 & 0.075 & 2.286 & 0.02 & 0.084 \\
SOCIAL -> PARALISAÇÃO & 3.163 & 0.007 & -0.137 & 0.084 & 1.639 & 0.10 & $* * *$ \\
AFETIVO -> SEGURANÇA & 2.212 & 0.000 & 0.030 & 0.062 & 0.483 & 0.63 & \\
COGNITIVO -> SEGURANÇA & 2.613 & 0.001 & 0.042 & 0.071 & 0.592 & 0.55 & 0.042 \\
SOCIAL -> SEGURANÇA & 3.163 & 0.009 & 0.164 & 0.076 & 2.160 & 0.03 & $* *$ \\
\hline
\end{tabular}

Nota: $(* *)$ significativo a 0,05 e $(* * *)$ significativo a 0,10 . 
Em relação às relações causais entre as dimensões e o tema "Paralisação dos eventos esportivos", pode-se observar pelo valor-p da estatística $t$ que somente o constructo Afetivo não foi significativo a $10 \%$ ( $p$-valor $<0,10)$. Dessa forma, as dimensões Cognitivos e Social influenciam a variável Paralisação. Essa relação é negativa, visto pelos valores do coeficiente. Quanto maiores os níveis Cognitivos e Sociais, menores serão as respostas para uma Paralisação dos eventos. O que é coerente, pois indivíduos que possuem necessidades de socializar fortemente com outras pessoas, seja por diferentes motivações, valores e até através do diálogo ou debate sobre o Esporte e seus eventos (PONS; MOURALI; NYECK, 2006; WANN et al., 2008), não gostariam da Paralisação dos mesmos. Apesar de que todos os tipos de fãs identificados tenderem para a concordância com relação à Paralisação dos eventos esportivos devido a pandemia COVID-19.

Para o tema de Segurança, os resultados foram diferentes. Somente a dimensão Social teve relação significativa com a variável Segurança, visto pelo valor-p inferior a 0,05. Além disso essa relação foi positiva, pelo coeficiente 0,164. 0 que indica que os níveis maiores para o comportamento Social respondem por mais segurança. $O$ que também faz sentido, já que para uma devida socialização é preciso aglomeração, interação e confraternização entre pessoas, o que não é possível neste momento de quarentena, dúvidas e angustia causados pela pandemia, como já apontado por alguns autores (GALLEGO et al., 2020; GUERREIRO et al., 2020; MOHR et al., 2020; TORESDAHL; ASIF, 2020).

É compreensível que a dimensão Afetiva não tenha tido significância para as Paralizações e Segurança, já que esse tipo de Orientação remete à fãs Experenciais, que são indivíduos que possuem como principal interesse a emoção de estar ou assistir o acontecimento esportivo (experiência) (PONS; MOURALI; NYECK, 2006). Ou seja, é incerto qual tipo de experiência que teremos no "novo normal", assim como é pouco certo o que será seguro e o que não será.

Por outro lado, será um desafio para as organizações responsáveis pelo Esporte em oferecer algo para seus fãs, no sentido que haverá provavelmente um forte desenvolvimento de transmissões esportivas, sem a presença de público in loco. O desafio na verdade é proporcionar uma Orientação, com experiências, socialização e debate em um "novo normal", e já existem opções para isso via streaming, redes sociais e outras ferramentas tecnológicos com relação a esta realidade (ROCCO JÚNIOR; MAZZEI, 2018; YANNIS et al., 2020).

Tais afirmações ainda são corroboradas com as respostas dos respondentes sobre, "caso os eventos esportivos voltem a ser realizados em 2020, qual o formato quanto a presença ou não de público?", conforme apresentado na Figura 4. 


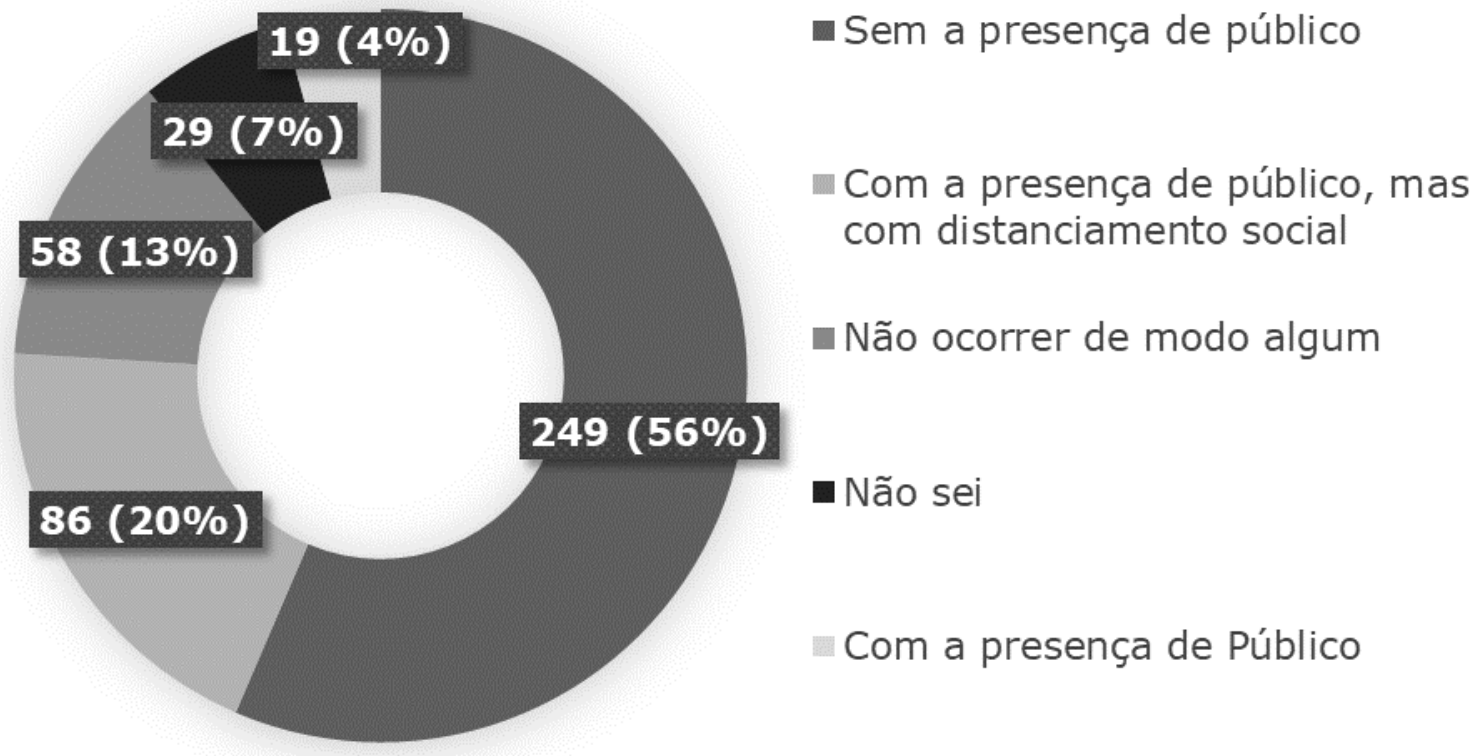

Figura 4 - Opinião dos respondentes quanto a presença de público em eventos esportivos a serem realizados em 2020 .

Sendo assim, de acordo coma opinião dos respondentes que participaram desta pesquisa, a presença de público em eventos esportivos até o final de 2020 está provavelmente comprometida, seja pela opinião dos mesmos, por razões de segurança e possivelmente em razão de normas sanitárias. Além disso, em meio ao grande momento de incertezas, as opiniões dos respondentes tendem a confirmar o resultado de outras pesquisas, ou seja, boa parte das pessoas não é favorável à realização de eventos esportivos em curto e médio prazos, o que corresponde ao mês de maio/junho de 2020 até dezembro/janeiro de 2021.

Como limitações, este artigo apresentou uma amostragem de certa forma limitada para o universo nacional. Ao mesmo tempo, as variáveis da dimensão "Cognitivo" devem ser revistas em uma amostragem maior, ou revistas quanto à sua redação, afim de confirmar a sua confiabilidade. Neste estudo, o Alfa de Cronbach para esta dimensão teve valor abaixo do recomendado pela literatura. Por outro lado, os resultados são semelhantes à outras pesquisas de opinião divulgadas na mídia por outros grupos científicos. Também há a limitação dos resultados perderem o sentido no momento que novos desdobramentos surgem com os contextos relacionados à crise pandêmica da COVID-19. Um tratamento efetivo ou vacina mudaria todo o cenário por exemplo.

\section{CONSIDERAÇÕES FINAIS}

O objetivo deste artigo foi realizar a tradução e a validação de uma escala relacionada com as Motivações e os Valores pessoais que levam cada indivíduo a se fazerem presentes em eventos esportivos, assim como um melhor 
entendimento sobre o comportamento do público com relação a pandemia do COVID-19 e os eventos esportivos, especificamente quanto a Paralisação e a Segurança. A partir dos resultados, que possuem embasamento e são até "complexos", percebe-se que a escala OSE, baseada no conceito de "Orientação" (Motivações somadas à Valores) é um valioso conceito para se analisar o comportamento do público para a presença de eventos esportivos.

Ao mesmo tempo, com relação aos eventos esportivos e a pandemia COVID19, conclui-se que há uma significância na relação entre o comportamento das pessoas que buscam os eventos para socializar (dimensão Social, Fãs Sociais) e o tema Paralisação dos eventos. Em tese, essas pessoas gostariam que os eventos não fossem paralisados, apesar de concordarem com a paralisação. A mesma característica acontece para o comportamento relacionado com a busca por boas experiências (dimensão Afetiva, Fãs Experenciais) e a Paralisação dos eventos esportivos. A dimensão relacionada com o perfil dos Fãs Sociais também demonstrou ter significância com a Segurança, e esta temática talvez seja imprescindível para a volta da realização de eventos esportivos. Conforme apresentado no decorrer deste artigo, a característica "socialização" parece ser inerente às necessidades, motivações e valores das pessoas que buscam os eventos esportivos. O que, consequentemente, permite prospectar que, com relação aos eventos esportivos, os impactos serão enormes, tanto econômicos, como sociais. Em contrapartida, existem algumas possibilidades para a realização dos eventos daqui em diante, considerando os desdobramentos da pandemia causada pela COVID-19. De fato, a socialização fica prejudica, apesar de ser uma situação visada pelo público. Mas, é possível criar e organizar eventos esportivos que contemplem outros comportamentos, como os do "Super fãs" através da utilização de ferramentas tecnológicas para uma transmissão ou envolvimento diferenciado do público (exemplo, corridas de rua virtuais), propor experiências diferenciadas e que priorizem a segurança (exemplo, engajamento remoto e sonorização de torcidas em jogos com transmissão pela mídia), dentre outros. Caberá aos organizadores uma maior capacidade de gestão para a realização de eventos esportivos.

Chegamos a uma consideração de que o esporte sempre foi um dos principais fenômenos sociais e uma das maiores instituições do mundo moderno. Como bem referenciado, ao longo dos dois últimos séculos ele refletiu e influenciou como a sociedade humana vem se organizando, espelhando as diferentes formas de estruturas entre Estados, povos e classes sociais, além de se tornar um dos principais elementos da indústria cultural e de entretenimento contemporânea. Tema constante para o diálogo cotidiano, matéria prima dos meios de comunicação e também uma forma reconhecida e valorizada para a ascensão social por aqueles que se dedicam à essa busca (RUBIO, 2002).

Entretanto, em 2020, o acontecimento pandêmico causado por um organismo acelular afeta e questiona de forma contundente sobre qual o Esporte 
vivíamos, e qual o Esporte que iremos ter no futuro. Como todos os fatos, o Esporte naturalmente se inseriu em discussões de inúmeras esferas, como a Saúde, a Econômica, a Política, a Social, dentre outras. Especificamente sobre os eventos esportivos, fica cada vez mais claro que ele é parte de um fenômeno, talvez uma parte que pode se dar ao luxo de ser preservada neste momento de crise mundial. O mais importante será a preservação de vidas de todos os envolvidos com a realização de eventos esportivos, como atletas e familiares, funcionários das organizações esportivas e familiares, árbitros e familiares, profissionais de mídia e familiares etc.

Chegou um momento de reflexão sobre a necessidade de ajuda de todos os envolvidos com o Esporte e seus eventos, desde as organizações responsáveis, o poder público, o setor privado, até a mídia que os transmite. Todos deverão buscar alternativas e soluções, principalmente para a ajuda aos menos favorecidos em termos socioeconômicos, que geralmente são os atletas e funcionários operacionais das organizações. Espera-se esperança e união. De qualquer forma, este artigo deixa como contribuição do conhecimento a utilização da escala OSE e o conceito de Orientação para eventos esportivos, que poderá ser utilizada em outros estudos com diferentes perspectivas, análises, constructos e/ou novos contextos.

\section{REFERÊNCIAS}

BEATON, Dorcas E.; BOMBARDIER, Claire; GUILLEMIN, Francis; FERRAZ, Marcos Bosi. Guidelines for the process of cross-cultural adaptation of self-report measures. Spine, $v$. 25, n. 24, p. 3186-91, 2000.

BÖHME, Maria Tereza Silveira. Esporte Infantojuvenil: Treinamento a Longo Prazo Teoria e Prática. São Paulo: Phorte, 2011.

BOUCHET, Patrick; BODET, Guillaume; BERNACHE-ASSOLLANT, Iouri; KADA, Faycel. Segmenting sport spectators: Construction and preliminary validation of the Sporting Event Experience Search (SEES) scale. Sport Management Review, v. 14, n. 1, p. 42-53, 2011.

BRUNER II, Gordon C. Marketing Scales Handbook: a Compilation of Multi-Item Measures for Consumer Behavior \& Advertising Research. Carbondale, Illinois: GCBII Productions, 2009.

CHEN, Huijun; GUO, Juanjuan; WANG, Chen; LUO, Fan; YU, Xuechen; ZHANG, Wei; LI, Jiafu; ZHAO, Dongchi; XU, Dan; GONG, Qing; LIAO, Jing; YANG, Huixia; HOU, Wei; ZHANG, Yuanzhen. Clinical characteristics and intrauterine vertical transmission potential of COVID-19 infection in nine pregnant women: a retrospective review of medical records. The Lancet, v. 395, n. 10226, p. 809-815, 2020.

CRESWELL, John W. Projeto de Pesquisa: Métodos Qualitativos, Quantitativo e Misto. 3. ed. Porto Alegre: Artmed, 2010.

DE BOSSCHER, Veerle; SHIBLI, Simon; WESTERBEEK, Hans; VAN BOTTENBURG, 
Maarten. Successful Elite Sport Policies: An international comparison of the SportsPolicy factors Leading to International Sporting Success (SPLISS 2.0) in 15 nations. Aachen: Meyer \& Meyer Verlag, 2015.

FUNK, Daniel; ALEXANDRIS, Kostas; MCDONALD, Heath. Sport Consumer Behaviour: Marketing Strategy. Oxon; New York: Routledge, 2016.

FUNK, Daniel C.; FILO, Kevin; BEATON, Anthony A.; PRITCHARD, Market. Measuring the Motives of Sport Event Attendance: Bridging the Academic-Practitioner Divide to Understanding Behavior. Sport Marketing Quarterly, v. 18, n. 3, p. 126-138, 2009.

GALLEGO, Viviana; NISHIURA, Hiroshi; SAH, Ranjit; RODRIGUEZ-MORALES, Alfonso J. The COVID-19 outbreak and implications for the Tokyo 2020 Summer Olympic Games. Travel Medicine and Infectious Disease, v. 34, p. 101604, 2020.

GAMMELSÆETER, Hallgeir. Sport is not industry: bringing sport back to sport management. European Sport Management Quarterly, p. 1-23, 2020.

GILLESPIE, Willian; MCDONALD, Mark. Gestão de Eventos Esportivos. In: VANCE, Patricia; NASSIF, Vânia; MASTERALEXIS, Lisa. Gestão do Esporte: casos brasileiros e internacionais. Rio de Janeiro: LTC-Grupo Gen, 2015. p. 217-241.

GUERREIRO, Renato Carvalho; SILVA, Andressa; ANDRADE, Henrique Araújo;

BIASIBETTI, Isadora Grade; VITAL, Roberto; SILVA, Hesojy Gley Vital; SILVA, Flavia Rodrigues; MELLO, Marco Túlio de. O adiamento dos Jogos Olímpicos e Paralímpicos de Tóquio 2020 foi uma decisão correta? Revista Brasileira de Medicina do Esporte, v. 26, n. 3, p. 277-282, 2020.

HAIR, Joseph F.; BLACK, William C.; BABIN, Barry J.; ANDERSON, Rolph E.; TATHAM, Ronald L. Análise multivariada de dados. 6. ed. Porto Alegre: Bookman, 2009.

HAIR, Joseph F.; SARSTEDT, Marko; HOPKINS, Lucas; KUPPELWIESER, Volker G. Partial least squares structural equation modeling (PLS-SEM). European Business Review, v. 26, n. 2 , p. $106-121,2014$

KASZNAR, Istvan K.; GRAÇA, Ary S. A Indústria do Esporte no Brasil. 2. ed. São Paulo: M.Books do Brasil Editora, 2012.

MALHOTRA, Naresh K. Pesquisa de Marketing: uma orientação aplicada. 6. ed. Porto Alegre: Bookman, 2011.

MASLOW, Abraham H. Introdução à Psicologia do Ser. 2. ed. Rio de Janeiro: Eldorado, 2016.

MASTERMAN, Guy. Strategic Sports Event Management. 3. ed. London; New York: Routledge, 2014.

MATTAR, Fauze Najib; MATTAR, Michel Fauze. Gestão de Negócios Esportivos. Rio de Janeiro: Elsevier, 2013.

MOHR, Magni; NASSIS, George P.; BRITO, Joao; RANDERS, Morten B.; CASTAGNA, Carlo; PARNELL, Dan; KRUSTRUP, Peter. Return to elite football after the COVID-19 lockdown. Managing Sport and Leisure, p. 1-9, 2020.

NICOLA, Maria; ALSAFI, Zaid; SOHRABI, Catrin; KERWAN, Ahmed; AL-JABIR, Ahmed; IOSIFIDIS, Christos; AGHA, Maliha; AGHA, Riaz. The socio-economic implications of the coronavirus pandemic (COVID-19): A review. International Journal of Surgery, v. 78, p. 
185-193, 2020.

PASQUALI, Luiz. Instrumentação Psicológica: fundamentos e práticas. Porto Alegre: Artmed, 2010.

PITTS, Brenda G.; FIELDING, Lawrence W.; MILLER, Lori K. Industry Segmentation Theory and the Sport Industry: Developing a Sport Industry Segment Model. Sport Marketing Quarterly, v. 3, n. 1, p. 15-24, 1994.

PLEWA, Carolin; PALMER, Karen. Self-congruence theory: towards a greater understanding of the global and malleable selves in a sports specific consumption context. International Journal of Sports Marketing and Sponsorship, v. 15, n. 4, p. 26$39,2014$.

POIT, Davi Rodrigues. Organização de Eventos Esportivos. 5. ed. São Paulo: Phorte Editora, 2013.

PONS, Frank; MOURALI, Mehdi; NYECK, Simon. Consumer Orientation Toward Sporting Events. Journal of Service Research, v. 8, n. 3, p. 276-287, 2006.

PREUSS, Holger. The Conceptualisation and Measurement of Mega Sport Event Legacies. Journal of Sport \& Tourism, v. 12, n. 3-4, p. 207-228, 2007.

ROCCO JÚNIOR, Ary José; MAZZEI, Leandro Carlos (Org.). Os Estádios e Arenas do Futebol Brasileiro e o legado da Copa do Mundo 2014: o padrão FIFA, o consumidor do esporte e o entretenimento. Sarapuí: OJM Casa Editorial, 2018.

RUBIO, Katia. Do Olimpo ao Pós-Olimpismo: Elementos para uma reflexão sobre o esporte atual. Revista Paulista de Educação Física, v. 16, n. 2, p. 130-143, 2002.

TORESDAHL, Brett G.; ASIF, Irfan M. Coronavirus Disease 2019 (COVID-19): Considerations for the Competitive Athlete. Sports Health: A Multidisciplinary Approach, v. 12, n. 3, p. 221-224, 2020.

WANN, Daniel; GRIEVE, Frederick G.; ZAPALAC, Ryan K.; PEASE, Dale G L. Motivational Profiles of Sport Fans of Different Sports. Sport Marketing Quarterly, v. 17, n. 1, p. 6-19, 2008.

YANNIS, Lianopoulos; THEODORAKIS, Nicholas D.; TSIGILIS, Nikolaos; GARDIKIOTIS, Antonis; KOUSTELIOS, Athanasios. Elevating self-esteem through sport team identification: a study about local and distant sport fans, 2020. ahead-of-print. 